\title{
Cosmic infrared background measurements and star formation history from Planck
}

\author{
Paolo Serra, on behalf of the Planck Collaboration \\ Institut d'Astrophysique Spatiale (IAS), \\ Bâtiment 121, F- 91405, Orsay (France) \\ email: pserra@ias.u-psud.fr
}

\begin{abstract}
We present new measurements of Cosmic Infrared Background (CIB) anisotropies using Planck. Combining HFI data with IRAS, the angular auto- and cross-frequency power spectrum is measured from 143 to $3000 \mathrm{GHz}$. After careful removal of the contaminants (cosmic microwave background anisotropies, Galactic dust and Sunyaev-Zeldovich emission), and a complete study of systematics, the CIB power spectrum is measured with unprecedented signal to noise ratio from angular multipoles $\ell \sim 150$ to 2500 . The interpretation based on the halo model is able to associate star-forming galaxies with dark matter halos and their subhalos, using a parametrized relation between the dust-processed infrared luminosity and (sub-)halo mass, and it allows to simultaneously fit all auto- and cross- power spectra very well. We find that the star formation history is well constrained up to redshifts around 2, and agrees with recent estimates of the obscured star-formation density using Spitzer and Herschel. However, at higher redshift, the accuracy of the star formation history measurement is strongly degraded by the uncertainty in the spectral energy distribution of CIB galaxies. We also find that the mean halo mass which is most efficient at hosting star formation is $\log \left(M_{\mathrm{eff}} / \mathrm{M}_{\odot}\right)=12.6$ and that CIB galaxies have warmer temperatures as redshift increases.
\end{abstract}

Keywords. galaxies: high-redshift, (cosmology:) dark matter, (cosmology:) diffuse radiation

\section{Introduction and data used}

The relic emission from galaxies formed throughout cosmic history appears as a diffuse, cosmic background. The Cosmic Infrared Background (CIB) is the far-infrared part of this emission and it contains about half of its total energy (Dole et al. 2006). The anisotropies detected in this background light trace the large-scale distribution of starforming galaxies and, to some extent, the underlying distribution of the dark matter halos in which galaxies reside. The CIB is thus a direct probe of the interplay between baryons and dark matter throughout cosmic time and it allows an accurate determination of the star formation history of the Universe, which has long been a fundamental goal in Astronomy. Combining Planck HFI data with IRAS, the CIB angular auto- and cross-frequency power spectra are measured from 143 to $3000 \mathrm{GHz}$ and they are interpreted with a Halo Model that associates star-forming galaxies with dark matter halos and their sub-halos, using a parametrized relation between the dust-processed infrared luminosity and (sub-)halo mass. The star formation history is well constrained up to redshifts around $\mathrm{z} \sim 2$, and agrees with recent estimates of the obscured star formation density using Spitzer and Herschel. However, at higher redshift, the accuracy of the star formation history measurement is strongly degraded by the uncertainty in the spectral energy distribution of CIB galaxies.

We use Planck channel maps in Healpix (Gorski et al. 2005) format from the six HFI frequencies: 100, 143, 217, 353, 545, and $857 \mathrm{GHz}$, together with far-infrared data at 3000 $\mathrm{GHz}(100 \mathrm{~m})$ from IRAS (IRIS, Miville-Deschenes \& Lagache 2005). Although Planck 


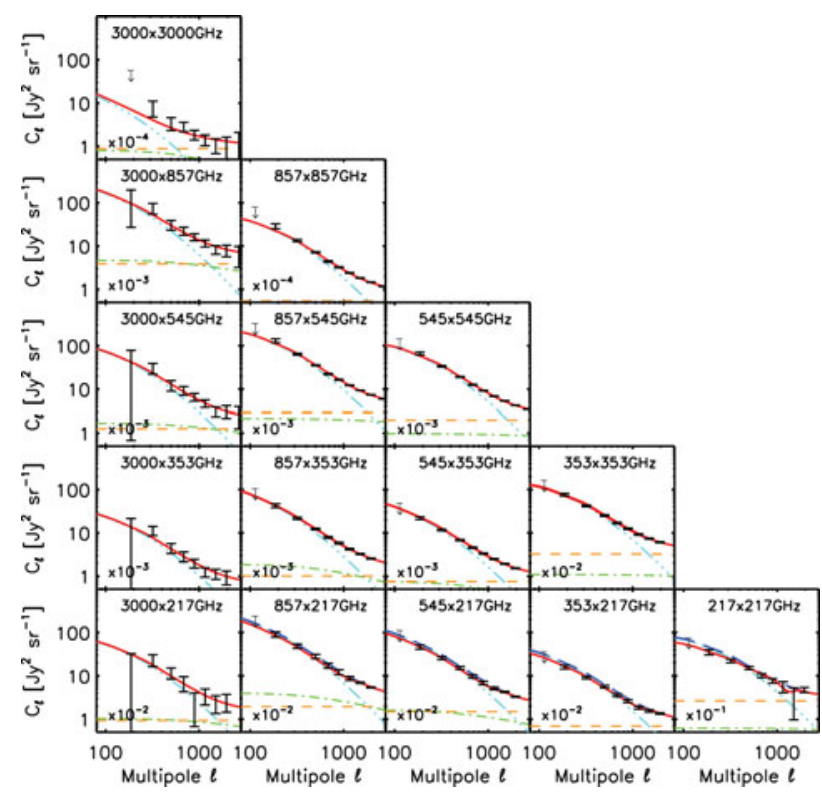

Figure 1. (Cross-) power spectra of the CIB anisotropies measured by Planck and IRAS, compared with the best-fit extended halo model. Data points are shown in black. The red line is the sum of the linear, 1-halo and shot-noise components, which is fitted to the data. The orange dashed, green dot-dashed, and cyan three-dots-dashed lines are the best-fit shot-noise level, the 1-halo and the 2-halo terms, respectively.

is an all-sky survey, we restricted our CIB anisotropy measurements to a few fields at high Galactic latitude, where foregrounds can be more easily controlled. The total area used to compute the CIB power spectrum is about $2240 \mathrm{deg}^{2}, 16$ times larger than in Planck Collaboration XVIII (2011). Measuring the CIB anisotropies is not easy, and it requires a very accurate component separation. Galactic dust, Cosmic Microwave Background (CMB) anisotropies, emission from galaxy clusters through the thermal SunyaevZeldovich (tSZ) effect, and point sources all have a part to play. To remove the CMB we use a simple subtraction technique, using the HFI lowest frequency channel $(100 \mathrm{GHz})$ as a CMB template and taking into account for its contamination due to CIB and tSZ residuals. For the Galactic dust we use an independent, external tracer of diffuse dust emission, the HI gas. From $100 \mu \mathrm{m}$ to $1 \mathrm{~mm}$ at high Galactic latitude and outside molecular clouds, a tight correlation is observed between far-infrared emission from dust and the 21-cm emission from gas: HI can thus be used as a tracer of cirrus emission and the dust can be removed accordingly.

\section{Results: constraints on Star Formation History}

With a best-fit $\chi^{2}$ of 100.7 and 98 degrees of freedom, we obtain a very good fit to the data (see Fig. 1) using an extended version of the standard halo model. The mean value of the most efficient halo mass for generating the CIB, $\log \left(\mathrm{M}_{\mathrm{eff}} / \mathrm{M}_{\odot}\right)=12.6 \pm 0.1$, is in good agreement with results obtained from a similar analysis using Herschel data. The star formation rate density (SFRD) predicted (see Fig. 2) is in agreement with other measurements up to redshift $\mathrm{z} \sim 2$, while at higher redshifts our model predicts higher values for the SFRD respect to other measurements (see e.g. Gruppioni et al. 2013) and also a different analysis of Planck data using only linear scales, i.e. multipoles $l<500$ ). 


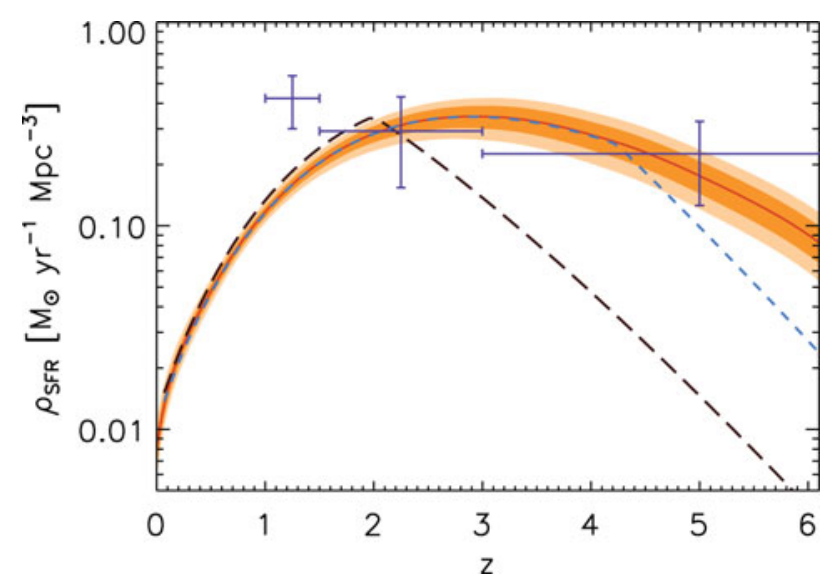

Figure 2. Marginalized constraints on the star formation rate density, as derived from our extended halo model (red continuous line with \pm 1 and $\pm 2 \sigma$ orange dashed areas). It is compared with mean values computed imposing different conditions on the redshift evolution of galaxy luminosities (black long-dashed line and blue dashed line). The violet points with error bars are the SFR density determined from the modeling of the CIB-CMB Lensing cross correlation by Planck 2013 Results XVIII.

However, the SFRD predicted is in very good agreement with the estimate obtained from the gravitational lensing-infrared background correlation with Planck data (see Planck Collaboration XVIII). Knowing that the SEDs of the galaxies responsible for the bulk of the CIB is the principal limitation in our modeling framework, the accurate measurement of the SEDs with future surveys will be crucial to properly estimate the obscured star formation rate density at high redshift from the CIB anisotropies and thus determine whether or not the bulk of the star formation is obscured at high redshift, and whether the UV and Lyman-break galaxy populations are a complete tracer of the star formation in the early Universe.

\section{Acknowledgments}

The development of Planck has been supported by: ESA; CNES and CNRS/INSUIN2P3-INP (France); ASI, CNR, and INAF (Italy); NASA and DoE (USA); STFC and UKSA (UK); CSIC, MICINN and JA (Spain); Tekes, AoF and CSC (Finland); DLR and MPG (Germany); CSA (Canada); DTU Space (Denmark); SER/SSO (Switzerland); RCN (Norway); SFI (Ireland); FCT/MCTES (Portugal); and PRACE (EU). A description of the Planck Collaboration and a list of its members with the technical or scientific activities they have been involved into, can be found at ESA's RSSD website. The Parkes radio telescope is part of the Australia Telescope National Facility which is funded by the Commonwealth of Australia for operation as a National Facility managed by CSIRO. Some data used in this paper are based on observations with the 100-m telescope of the MPIfR (Max-Planck-Institut fur Radioastronomie) at Effelsberg.

\section{References}

Dole, H., Lagache, G., Puget, J. L., et al. 2006, A\& $A$, 451, 417

Gorski, K. M., Hivon, E., Banday, A. J., et al. 2005, ApJ, 622, 759

Gruppioni, C., Pozzi, F., Rodighiero, G. et al.. 2013, MNRAS, Vol. 432, 23-52

Miville-Deschenes, M. -A. \& Lagache, G. 2005, ApJS, 157, 302

Planck Collaboration XVIII 2013, accepted for publication in $A \& A$ 